\title{
The Role of Relaxation Therapy and Cranial Electrotherapy Stimulation in the Management of Dental Anxiety in Nigeria
}

\author{
Koleoso $\mathrm{ON}^{1}$, Osinowo $\mathrm{HO}^{2}$, Akhigbe $\mathrm{KO}^{3}$ \\ ${ }^{1}$ (Department of Mental Health, University of Benin Teaching Hospital, Benin City, Nigeria) \\ ${ }^{2}$ (Department of Psychology, University of Ibadan, Ibadan, Nigeria) \\ ${ }^{3}$ (Department of Mental Health, University of Benin Teaching Hospital, Benin City, Nigeria)
}

\begin{abstract}
Dental anxiety has been managed using pharmacological techniques in clinical situations. These treatment methods are not without side effects which sometimes could be hazardous. The present study assessed the effectiveness of relaxation therapy, and cranial electrotherapy stimulation (CES) in the treatment of dental anxiety. In this study, 40 respondents (Mean Age $=33.05$; $S D=11.12$ ) who scored high on dental anxiety ( $\bar{x}$ $=14.25)$ participated in the intervention programmes. Ten respondents were assigned to each of the control and experimental groups (Control $=10$, Relaxation group $=10$, CES group $=10$, Combined treatment group $=10$ ). Two hypotheses were tested in the study. Percentages and means, the t-test for independent samples, and one way ANOVA were used for analyses. Results show that exposure to therapy significantly reduced dental anxiety at post- test than at the pre-test $(t(164)=11.33, p<.01)$. Respondents who were exposed to relaxation therapy $(\bar{x}=10.70 ; p<.05)$, CES treatment $(\bar{x}=10.20 ; p<.05)$, and the combined treatment $(\bar{x}=9.40 ; p<.05)$ reported significantly lower dental anxiety compared to those in the control group $(\bar{x}=18.30)$. In conclusion, the combination of relaxation and cranial electrotherapy stimulation significantly reduced dental anxiety and found beneficial for alleviating dental anxiety among dental patients.
\end{abstract}

Keywords:Cranial electrotherapy stimulation, Dental anxiety, Oral pain perception, Relaxation therapy, Nigeria

\section{Introduction}

The fear of dental treatment continues to be an issue within dentistry to the extent that about $31 \%$ of adults express this feeling. The attendant anxiety and fear of dental treatment prevent sufferers from seeking dental care $[\mathbf{1 , 2}]$. When such individuals manage to actually seek care, they often become difficult to manage once they are in the dental chair. There have been some occasions when anxious patients in the dentist's office reported experiences of pain, and ineffective local anaesthesia [3,4]. Anxiety associated with dental care interferes with the provision of oral healthcare and can cause subjects living with oral pain to postpone, or even keep away from essential care altogether [5]. Preceding research has recognized that high levels of dental anxiety are related to longer intervals between dental visits, poorer oral function and a high frequency of oral symptoms [6]. These research findings indicate the importance of addressing people who are living with oral pain conditions.

Alleviating patient anxiety has been a worry in the dental profession. At the moment, dentists have a range of modalities existing to decrease patients' anxiety. Good examples include medication, electronic anaesthesia, acupuncture, hypnosis, air-abrasion dental hand pieces, and nitrous oxide. Each one has its benefits and difficulties. Concerning the drawbacks, some are very expensive, some are too time- consuming, and some have a long learning curve. Others are limited by patients' medical conditions, or have persistent side effects after management [7].Hence the need to introduce non pharmacological procedures in the treatment of anxiety related to dental care. Current investigation advocates that the most valuable outcome of relaxation training procedures is the ability to obtain deep muscle relaxation [8]. It has also been known for some time that electrical stimulation affects physiological changes and cranial electrotherapy stimulation (CES) technology appears to offer an easy to use, safe and cost effective treatment to alleviateanxiety[7]. Previous research has revealed that CES was successful, the same as relaxation therapy in reducing anxiety, but easier to administer [9].

Aartman, de Jongh, Makkes, and Hoogstraten[10] argue that behavioural management seems to be superior to anxiolytic drug therapy. Halvorsen and Willumsen[11] note that dentally anxious patients reported that they prefer non-pharmacological interventions. Most of the behaviourally-oriented treatments include components based on systematic desensitization and use of relaxation to counteract and weaken the fear response during gradual exposure to treatment [12]. Berggren, Hakeberg, and Carlsson[13] conducted a study in which training in progressive muscular relaxation led to a greater reduction in anxiety among dentally anxious patients than did a cognitive approach. A possible explanation for this is that the perception of personal control achieved with applied relaxation, is a clinically important factor that influences patient's level of acute pain, 
which is known to be a primary source of dental anxiety during stressful dental procedure [14]. Thus, the question arises as to whether interventions aimed at patients' psycho-physiological arousal, such as relaxation techniques, result in effects that are different from those achieved with approaches that involve more passive distraction from the anxiety-provoking stimuli [15].

Lahmann et al. [15] investigated the effectiveness of two interventions, viz, Brief Relaxation and Music Distraction, in reducing dental anxiety in comparison with no intervention. The results show that brief relaxation was more effective than music distraction. Although music distraction also proved to be beneficial in reducing state anxiety in comparison with no intervention, the effect sizes were moderate $[\mathbf{1 6}, \mathbf{1 7}]$. While the effect of brief relaxation was greater among highly anxious respondents, musical distraction demonstrated its greatest effect among respondents with moderate anxiety [15].Berggren,Hakeberg, \&Carlsson[13] investigated the outcome of two different methods for the treatment of dental fear: relaxation and cognitively-oriented therapy. It was shown that, while cognitively-oriented therapy resulted in a higher number of patients completing therapy, relaxation-oriented treatment generally resulted in a more significant reduction in outcome measures of dental fear as well as in general anxiety and fear.

Over the years, transcutaneous electrical nerve stimulation has become widely accepted by physicians and dentists as a means of controlling many forms of pain [7]. Alpha-Stim (Electromedical Products International Inc, Mineral Wells, TX) cranial electrotherapy stimulation (CES) technology has been found in to be effective in long-term phobic patients to the 0.0001 level of confidence [18]. In a series of electroencephalographic studies, Heffernan [19] showed spectral smoothing consistent with pain reduction from this modality.

Winick[7] evaluates CES for dental anxiety in anticipation of, during, and at the conclusion of various routine dental procedures and found that patients who experience anxiety are significantly comforted during various dental procedures through the use of CES. The results indicate a very significant improvement in patients' levels of anxiety at the completion of the procedure. Many members of the treatment group requested CES at subsequent visits, and none objected to it. Cranial Electrotherapy Stimulation (CES) is a non-invasive technique used for treating various conditions [20]. The analgesic action of sub-perception levels of CES has been demonstrated in various clinical pain models [21]. Extracellular recording techniques indicated that CES modifies noxious stimuli-evoked responses in the regions of the rat brain that are involved in nociceptive processing [22].

In humans, the mechanism of action of CES is not fully understood. However, it has been shown to stabilize neurotransmitter turnover [23]. According to Gold, Pottash, Sternbach, Barbaban, and Asunitto[24], it stimulates production of insulin growth factor -1 , and facilitates normalization of monoamine levels following experimentally induced noxious stress. In addition, CES has been shown by Stanley, Cazalaa, Limoge, and Louville[25] to enhance anaesthetic effects in humans. They reported that CES increased nitrous oxide potency by approximately 37 percent, and noted that it reduced the required analgesic dose of Afentanyl by approximately 33 percent in patients undergoing urologic surgery.Braverman, Smith, Smayda, and Blum [26] found that CES also has anxiolytic-enhancing effects, while Philip, Demotes-Mainard, Bourgeois, and Vincent [27] discovered mood-enhancing effects in humans. Kulkarni and Smith [28] demonstrated CES to effectively decrease spinal spasm. In a related research, Romano [29] found it to effectively reduce headache spasm. Hochman[30] shows that it can alleviate dental spasm, while Kirsch and Lerner [31] demonstrated its effectiveness in reducing muscle pain spasm as well as control several conditions often associated with pain (for example anxiety, depression, insomnia, and generalized stress) [32].

In two post-marketing surveys, 47 physicians reported the results of CES use by 500 patients for various conditions [33]. Six patients $(1.2 \%)$ reported dizziness and two $(0.4 \%)$ reported nausea, both of which normally occur if the current is set too high. Three patients $(0.6 \%)$ reported skin irritation, and one each $(0.2 \%)$ reported anger, a metallic taste, a heavy feeling, and intensified tinnitus. Smith [34] reported that out of 23 psychiatric outpatients, $1(4.3 \%)$ cried during treatment and $1(4.3 \%)$ reported skin irritation behind the ears when the electrodes gel began drying out. Tan et al. [20] claim that such side effects appear to be related to the use of higher voltages and the placement of electrodes on the eyes.

Several studies conducted during the 1990 s, with a total of 259 respondents, reported no side effects that could reasonably be attributed to CES use [35]. To clarify the diverse published results of cranial, electro stimulation (CES) efficacy, Klawansky, Yeung, Berkey, Shan, Phan, and Chalmers [36] conducted an extensive literature review that identified eight of the most carefully conducted randomized controlled trials of CES versus sham treatment. The meta-analysis of anxiety showed CES to be significantly more effective than sham. The authors also noted that improvement occurred in 7 of the 8 studies for anxiety that used continuous measurement scale.

Bianco[37] avers that the active CES, when combined with the normal treatment regimen given at the treatment facilities, was more effective in reducing anxiety and depression than the normal treatment regimen 
alone and the sham CES plus normal treatment regimen. Thus, the anticipated results regarding CES were supported, while the anticipated result regarding placebo effect was not supported. No side effect was reported.

In a retrospective study to determine the effect of cranial electrotherapy stimulation on patients suffering from anxiety disorders, Overcash [38] found that, during $6-8$ month follow-up, $73 \%$ of the patients were 'well satisfied' with their treatment and had no significant regression or other anxiety disorders, $18 \%$ were 'satisfied' but had some problems with anxiety since they stopped the treatment, and $9 \%$ that chose not to respond, had significant symptoms since they stopped the treatment, or in 1 case, 'was not satisfied' with the treatment. There was no reported side effect (short-or long-term). He claims that the results appear to be immediate in most cases, and that the CES treatment approach seems to have long-lasting results as long as the patient is given a minimum of $4-6$ treatments.

CES was also investigated among patients suffering from acute anxiety disorders. Overcash [35] reported a significant reduction in anxiety in both physiological objective measures and subjective measures in the patients that received CES treatment. Their average perceived anxiety was reduced to a normal level. There was an $86 \%$ correlation between the perceived anxiety ratings and the objective measures, indicating that, at least, with these patients, the subjective measures were relatively accurate assessments.

The use of relaxation therapy and cranial electrotherapy stimulation (CES) in the management of dental anxiety in this environment is not clear. Traditionally, dental anxiety has been managed using pharmacological techniques. Benzodiazepines are the most frequently used drugs in clinical situations associated with anxiety and panic disorders [39]. These drugs have dangerous side effects, including accidental, intentional and/or suicidal overdosing. Therefore, dental anxiety seems to be a good model for studying a potentially anxietyreducing procedure such as relaxation therapy and cranial electrotherapy stimulation (CES). Thus, examining the three non-pharmacological treatment modalities in reducing dental anxiety, that is relaxation therapy, cranial electrotherapy stimulation (CES), and combination of relaxation therapy and CES treatment is the focus of this study.

The review of literature therefore generated two hypotheses: there will be a significant reduction in dental anxiety at post- test than at pre- test among the respondents; and that respondents in the control group will score significantly higher dental anxiety than those in the relaxation therapy group, CES treatment group, and both treatments group.

\subsection{Research Design}

\section{Materials and Method}

This is a quasi-experimental research utilizing a pre-post completely randomized design. This study assessed the therapeutic efficacy of three treatment modalities which include relaxation therapy, cranial electrotherapy stimulation (CES) and combination treatment of relaxation and CES treatment on dental anxiety. A baseline was obtained, and then the intervention programmes were introduced to the respondents after which a post- test data was collected. This was used to establish the efficacy of the three therapeutic techniques.

\subsection{Setting and Participants}

The setting for this study was the Dental Centre of University of Benin Teaching Hospital, Benin City, Nigeria.Ethical approval was obtained to carry out this research from the Ethics and Research Committee of the Teaching Hospital. Consent was obtained from the participants after due explanation about the study was given then. The population consists of respondents who were experiencing oral pain conditions for not less than three months, scored high on dental anxiety scale and met the under listed inclusion and exclusion criteria.

The inclusion criteria were: (a) Respondents must have been clinically diagnosed chronic or acute on chronic oral pain individual (b) Pain due to identifiable physical oral pathology except oral cancer, (c) Respondents must be 18 years and above, and (d) Respondents with high dental anxiety score (Modified DAS $\geq$ 14.25)

Criteria for Exclusion were: (a) Facial Pain, (b) Acute oral pain less than three months, (c) Psychogenic oral pain, (d) Pain due to oral cancer, (e) Traumatic injuries associated with oral pain less than three months, and (d) People with gross mental abnormality or other diagnosable neurological disorders

\subsection{Instrument employed in the data collection}

A two-part questionnaire was used to collect data for the study.The first part contains items to measure the demographic information required in the study, which include personal data, gender, age, marital status, employment status, educational level, pain site, duration of the pain and so on.

The second part of the questionnaire contains items that measures dental anxiety using the popular Modified Dental Anxiety Scale (MDAS)[40]. MDAS consists of five items with a five-point Likert response for each question (score $1-5$ ). These questions relate to how respondents would feel 'if they had to go to the dentist tomorrow', 'waiting in the dentist's office in the dentist's chair', 'waiting while s/he gets the drill ready' 
and 'in the dentist's chair to have teeth cleaned'. The MDAS has an extra item about the respondent's anxietyto have a local anesthetic injection in the gum, above an upper back tooth'. Modified dental anxiety score can range from 5to 25. Newton and Edward [41] claim that the MDAS is highly internally consistent (Cronbach alpha $=0.93$ ) and shows good reliability over time (intra-class correlation coefficient $=0.93$ ). The MDAS was found to discriminate between the three groups of participants defined by self-reported anxiety levels, and correlated highly with the Spielberger Trait Anxiety Inventory [41]. In the present study,an alpha coefficient of .76 and a Spearman Brown equal length coefficient of .75 were obtained.

\subsection{Procedure}

The preliminary pilot study undertaken before the main study had established the mean for dental anxiety to be 14.25 . Thus, respondents who reported subjective high dental anxiety (Modified DAS $\geq 14.25$ ) after psychological assessment on the screening days of Mondays and Tuesdays were considered eligible to participate in the therapeutic intervention program on Wednesdays, Thursdays, and Fridays of the same week.

In this study, out of the 255 respondents who completed the questionnaire, one hundred and thirty-eight (138) were found to have reported subjective high dental anxiety based on their high score on the modified Dental Anxiety Scale. Of this number, twenty-two (22) said they would not be able to come for the next three days while seventy-six (76) others either refused to show up for the treatments or started but did not complete the three (3) sessions. The reason given by the twenty-two (22) respondents for not wishing to participate had to do with inadequate time and the fact that they were not resident in Benin. However, 40 respondents who reported subjective high dental anxiety (Modified DAS $\geq 14.25$ ) successfully completed the three (3) treatment sessions.

The respondents who reported subjective high dental anxiety and who agreed to come for the therapeutic interventions for the next three days were randomly allotted to one of the four groups (relaxation therapy group, CES treatment group, both treatments simultaneously group, and the no treatment control group) based on the order in which they were arriving back at the psychological assessment room after they had been treated by the dentist.

Treatment sessions for relaxation therapy involved respondents listening to 30 minutes of relaxation instructions that was played on an MP-3 audio recorder via an ear phone while CES treatment was given with Alpha -Stim 100 device. It was set at $0.5 \mathrm{~Hz}$ frequency, which produces a variable pulse width in this device. The current intensity of the CES was regulated, depending on the subject by turning the current up until respondents began to feel a bit light headed and then turned it down to their comfort level. The current was applied by ear clip electrodes in the treatment of dental anxiety for 45 minutes treatment in each session. The intensities that can be used ranges from 100 to 600 microamperes, and was often varied from day to day for some respondents. Treatment sessions for all respondents with high dental anxiety took place in the same room between 9am and 12noon each day. Each participant had an individual session with the lead author [9]. After the initial 3 days treatment, each participant, including those in Group D (control group) returned to the Dental Centre the following Monday to complete a post-intervention assessment in dental anxiety.

\subsection{Statistics and data analysis}

Responses to the questionnaires were coded and entered into the SPSS (version 15.0) for analysis. Apart from descriptive statistics, the analyses included: (1) reliability assessment of the independent and dependent scales (Cronbach alpha and split-half method); (2) t-test for independent sample used to compare the mean score of dental anxiety at pre-test and at post-test; and (3) one-way ANOVA used to compare dental anxiety on the no treatment control procedure and the basis of the application of treatment procedures.

\section{Results}

The responses of the respondents were subjected to a $t$-test for independent sample with pre-test and at post-test as independent variables and dental anxiety as dependent variable. Results of the analysis are presented in Table 1.

Table 1: Summary of Table of Independent T-Test Results Showing the Significant Influence of Treatment Procedures on Dental Anxiety

\begin{tabular}{|c|c|c|c|c|c|c|c|c|}
\hline Dependent Variable & Groups & Levels & $\mathbf{N}$ & $\mathbf{X}$ & S.D & $\mathbf{T}$ & D.F & $\mathbf{P}$ \\
\hline \multirow[b]{2}{*}{ Dental Anxiety } & & Pre-test & 40 & 17.93 & 2.70 & \multirow[b]{2}{*}{11.33} & \multirow[b]{2}{*}{39} & \multirow[b]{2}{*}{.05} \\
\hline & $\begin{array}{l}\text { Treatment } \\
\text { procedure }\end{array}$ & Post - test & 40 & 12.20 & 4.17 & & & \\
\hline
\end{tabular}

The result obtained show that treatment procedures significantly influenced dental anxiety $\mathrm{t}(164)=$ $11.33, \mathrm{p}<.01$. As predicted, there was a significant reduction in dental anxiety at post- test $(\bar{x}=12.20)$ than at pre-test $(\bar{x}=17.93)$ after the therapeutic interventions. 
To test the second hypothesis stated, a one-way analysis of variance (ANOVA) was carried out and the results are presented in Table 2 .

Table 2: One-Way ANOVA for Dental Anxiety among the Four Experimental Groups

\begin{tabular}{|lllllll|}
\hline Dependent Variable & Sources & SS & df & MS & F & P \\
& Between & 537.80 & 3 & 179.27 & & 45.90 \\
Dental Anxiety & Within & 140.60 & 36 & 3.91 & $<.01$ \\
& Total & 678.40 & 39 & & & \\
\hline
\end{tabular}

The results indicate that there was a significant difference between the control group and each of the active treatment group (relaxation therapy, CES treatment, and both treatments simultaneously) on dental anxiety $(\mathrm{F}[3,36]=45.90, \mathrm{p}<.01)$.

To determine the direction of significant difference, a post hoc test (Scheffe's) was carried out for dental anxiety as shown in Table 3 .

Table 3: Summary of Post Hoc Test Showing Difference between the Mean Scores of the Various Therapeutic Intervention Group on Dental Anxiety

\begin{tabular}{|c|c|c|c|c|c|c|c|}
\hline \multirow{3}{*}{$\begin{array}{l}\text { Dependent } \\
\text { Variable }\end{array}$} & Intervention Programmes & 1 & 2 & 3 & $\mathbf{X}$ & SD & $\mathbf{N}$ \\
\hline & CES Grp & & & & 10.20 & 1.48 & 10 \\
\hline & REL Grp & -.50 & & & 10.70 & 1.16 & 10 \\
\hline Dental & CES/REL Grp & .80 & 1.30 & & 9.40 & .97 & 10 \\
\hline Anxiety & CONTROL Grp & $-8.30^{*}$ & $-7.80^{*}$ & $9.10^{*}$ & 18.50 & 3.34 & 10 \\
\hline
\end{tabular}

$* \mathrm{p}<.05$

The result of the post hoc test shows that the mean score of respondents in the control group $(\bar{x}=$ 18.30) was significantly higher on dental anxiety than those reported by each of the active treatment groups (relaxation therapy group, $\bar{x}=10.70$; CES treatment group, $\bar{x}=10.20$; and CES/relaxation treatment group, $\bar{x}$ $=9.40$ ). However, the mean scores for the three active experimental groups: relaxation therapy group, CES treatment group, and CES/relaxation group, were not statistically different from one another on dental anxiety. Therefore, this hypothesis, which claims that the respondents in the control group will score significantly higher dental anxiety than those in the relaxation therapy group, CES treatment group, and both treatments group, is confirmed.

\section{Discussion}

This study revealed that treatment procedures significantly influenced dental anxiety with a significant reduction in dental anxiety at post- test than at pre-test after the therapeutic interventions. This finding suggests that the effect of treatment procedures (relaxation therapy, CES treatment, both treatment simultaneously) on dental anxiety or exposure to therapy significantly reduced dental anxiety at post-test than at the pre-test. This finding corroborates the findings of Aartman et al. [10], who claim that behavioural management seems to be superior to anxiolytic drug therapy. In a similar finding, Halvorsen and Wilumsen [42] noted that dentally anxious patients prefer non-pharmacological interventions. The hypothesized relationship was informed by the backdrop of numerous researchers who have for many years' explored non-pharmacological methods for use in the treatment of dental anxiety.

The study also revealed that there was a significant difference between the no treatment control group and each of the three active treatment groups (relaxation therapy group, CES treatment group, and CES/relaxation group) on dental anxiety. This result indicates that respondents in the control group were significantly higher on dental anxiety than those in each of the active treatment group. It was further noted that none of the three active treatment procedures proved to be significantly more effective than the other two. In line with the finding from this study, Hmud and Walsh [43] claim that relaxation techniques are safe, have no side effects, and give patients more control over their anxiety level. They went further to state that this treatment technique relaxes patients by reducing physical (muscle) tension, and makes dentally anxious patients more conscious of their stressed and aroused conditions, and enhance their ability to deal with these conditions. Besides, some studies indicate that general relaxation procedures can be just as effective as biofeedback [44]. This finding also corroborates the findings of Smith [34] which claimed that $86 \%$ of the respondents who were treated with CES showed remarkable improvements in their state anxiety and $90 \%$ in trait anxiety. In a similar study, Bystritsky Alexander, Kerwin, and Feusner[45] found that CES reduced the symptom burden of generalized anxiety disorder. The score on Hamilton Anxiety Rating Scale (HARS) was significantly reduced when the sampled population was assessed after 6 weeks. A meta-analysis of eight randomly controlled trial studies assessing the efficacy of CES on anxiety found that CES improved anxiety significantly as compared 
with placebo/sham treatment [46]. Confirming the result of this study, Zaghi, Acar, Hultgren, Boggio and Fregni[47] assert that CES increases the production of serotonin, GABA, and endorphins. These neurochemical changes may have accounted for any positive effects being experienced as a result of the use of CES.

Regardless of the specific mechanisms, the results of this study indicate that the CES treatment may be a useful adjunctive therapy for short-term treatment of symptoms of anxiety. The treatment appears to have about the same efficacy as the same amount of time of relaxation instructions, but it is easier to administer [9]. The present study shows, however, that relaxation therapy and CES can stand together or alone as significant, drug-free treatments for otherwise intractable dental anxiety.

\section{Conclusion}

This study has demonstrated the effectiveness of relaxation therapy and cranial electrotherapy stimulation and both treatments simultaneously in the management of dental anxiety. The implications are that non-pharmacological methods can be used in the treatment of dental anxiety. There are a number of treatment procedures that can be used by dentists for the alleviation of fear and anxiety in the dental office. At an intuitive level, many dentists probably use them very frequently as a comprehensive part of the everyday praxis. There are many areas in dentistry in which mental health professionals can make a significant contribution, an obvious one being the treatment and management of anxious patients using relaxation therapy and cranial electrotherapy stimulation. Considering the high number of fearful individuals visiting dentists regularly, a better knowledge about such treatment methods, which have been demonstrated in this study, would improve the dental care for the majority of these patients. It would also help prevent aggravation of fears among individuals at risk. Thus, there is the need for an improved education in behavioural dentistry. With proper education of health care professionals, it is easy enough to offer both treatments in a dental clinic.

However, despite the success of treatment methods performed by specially trained dentists, reported on by Aartman (2000), it seems reasonable that there should be limits to what can be expected of a dentist in terms of psychological diagnostic and therapeutic competence. Dental phobia may constitute a complex psychological and odontological problem with far-reaching consequences for a relatively large proportion of fearful individuals. Therefore, it seems likely that optimal care of such patients can best be achieved by crossdisciplinary efforts, involving dentists, psychiatrists and psychologists. The present study shows, however, that relaxation therapy and CES treatment can stand together or alone as significant, drug-free treatments for dental anxiety in the present study.

\section{Acknowledgements}

The authors wish to acknowledge the contributions of the patients and staff who took part in this study.

\section{References}

[1]. McGoldrick, P., de Jongh, A., \& Durham, R. (2001). Psychotherapy for dental anxiety. Cochjraze Database System Review, 2, CD 003070

[2]. Udoye, C. I., Oginni, A. O. \&Oginni, F. O. (2005). Dental anxiety among patients undergoing various dental treatments in a Nigeria teaching hospital. Journal of Contemporary Dental Practice. 6, 091-098.

[3]. Fiset, L., Milgrom, P., Weinstein, P., \&Melnick, S. (1989). Common fears and their relationship to dental fear and utilization of the dentist. Anesthetic Program. 36, 258-264.

[4]. Vassend, O. (1993). Anxiety, pain and discomfort associated with dental treatment. Behaviour Research and Therapy, $31,659-666$.

[5]. Gadbury-Amyot, C. \& William, K. B. (2000). Dental hygiene fear: Gender and age difference. Journal of Contemporary Dental Practices, 1, 1-11.

[6]. Hagglin, C., Beggren, U., Hakeberg, M. \&Ahlqwist, M. (1996). Dental anxiety among middle-aged and elderly women in Sweden. A study of oral state, utilisation of dental services and concomitant factors. Gerodontology, 13, 25-34

[7]. Winick, R. L. (1999). Cranial electrotherapy stimulation (CES): A safe and effective low cost means of anxiety control in a dental practice. Pain Control, 50-55.

[8]. Cott, A.(1981).The long term therapeutic significance of the addition of electromyographic biofeedback to relaxation training in the treatment of tension headaches. Behaviour Therapy, 12, 556-559.

[9]. Gibson, T. H. \& O' Hair, D. E. (1987). Cranial application of low level transcranial electrotherapy Vs. Relaxation instruction in anxious patients. American Journal of Electronmedicine, 4, 18-21.

[10]. Aartman, I. H. (2000). Treating highly anxious dental patients in a dental fear clinic. Academic Centre for Dentistry Amsterdam (ACTA). Amsterdam: Thesis.

[11]. Halvorsen, B. \&Willumsen, T. (2004). Willingness to pay for dental fear treatment is supplying dental fear treatment socially beneficial? European Journal of Health Economic, 5, 299-308.

[12]. Kvale, G., Berggren, U., \&Milgrom, P. (2004). Dental fear in adults: a meta-analysis of behavioural interventions. Community Dentistry and Oral Epidemiology, 32, 250-264.

[13]. Berggren, U., Hakeberg, M., \&Carlsson, S. (2000). Relaxation vs. Cognitive Oriented Therapies for Dental Fear. Journal of Dental Research, 79, 1645-1651.

[14]. Logan, H., Baron, R. S., Keeley, K., Law, A., \& Stein, S. (1991). Desired control and felt control as mediators of stress in a dental setting. Health Psychology, 10, 352-359.

[15]. Lahmann, C., Schoen, R., Henningsen, P., Ronels, J., Muehlbacher, M., Loew, T., Tritt, K., Nickel, M., \&Doering, S. (2008). Brief Relaxation versus Music Distraction in the Treatment of Dental Anxiety. Journal of American Dental Association, 139, $317-324$.

[16]. Muellner, M. (2002). Evidence based medicine. New York City: Springer. 
[17]. Peacock, J. \& Kerry, S. M. (2007). Presenting medical statistics: From proposal to publication. New York City: Oxford University Press.

[18]. Smith, R. B. \&Shiromoto, F. N. (1992). The use of cranial electrotherapy stimulation to block fear perception in phobic patients. Journal of Current Therapy and Research, 51, 249-253.

[19]. Heffernan, M. (1997). The effect of variable microcurrents on EEG spectrum and pain control. Canadian Journal of Clinical Medicine. 4, 4-11.

[20]. Tan, G., Rintala, D. H., Thornby, J., Yang, J., Wade, W., \&Vasilev, C. (2006). Using cranial electrotherapy stimulation to treat pain associated with spinal cord injury. Journal of Rehabilitation Research and Development, 43, 461-474.

[21]. Capel, I. D., Dorrell, H. M., \& Spencer, E. P. (1990). The application of sub-perception electrical stimuli elicits a temporally distinct response from restraint stress: 1. Antinociceptive characteristics. Journal of Bioelectricity, 9, 167-176.

[22]. Dong, W. O., Wilson, O. B., Skolnick, M. H., \&Dafny, N. (1992). Hypothalamic, dorsal raphe and external electrical stimulation modulate noxious evoke responses of habenula neurons. Neuroscience, 48, 933-940.

[23]. Poozos, R. S. Strack, L. E., White, R. K., \& Richardson, A. W. (1971). Electrosleep versus electroconvulsive therapy. In: Reynolds D. V. \&Sjoberg, A. E. (eds.). Neuroelectric Research. Springfield (IL): Charles Thomas.

[24]. Gold, M. S., Pottash, A. L. C., Sternbach, H., Barbaban, J., \&Asunitto, W. (1982). Anti-withdrawal effect of alpha methyl dopa and cranial electrotherapy. Presented at the Annual Meeting of the Society for Neuroscience; October 31 - November 5; Minneapolis, MN.

[25]. Stanley, T. H., Cazalaa, J. A., Atinault, A., Coeytaux, R., Limoge, A., \&Louville, Y. (1982). Transcutaneous cranial electrical stimulation decrease narcotic requirements during neuroleptanesthesia and operation in man Anesthesia and Analgesia, 61, 863866.

[26]. Braverman, E. Smith, R., Smayda, R., \& Blum, K. (1990). Modification of P300 amplitude and other electrophysiological parameters of drug abuse by cranial electrical stimulation. Current Therapy and Research, 48, 586-596.

[27]. Philips, P. Demotes-Mainard, J., Bourgeois, M., \& Vincent, J. D. (1991). Efficacy of transcranialelectrostimulation on anxiety and insomnia symptoms during a washout period in depressed patients; a double-blind study. Biological Psychiatry, 29, 451-456.

[28]. Kulkarni, A. D. \& Smith, R. B. (2001). The use of micro current electrical therapy and cranial electrotherapy stimulation in pain control. Clinical Practice of Alternative Medicine, 2, 99-103.

[29]. Romano, T. J. (1993). The usefulness of cranial electrotherapy in the treatment of headache in fibromyalgia patients. American Journal of Pain Management, 3, $15-19$.

[30]. Hochman, R. (1988). Neurotransmitter modulator (TENS) for control of dental operative pain. Journal of American Dental Association, 166, 208-212.

[31]. Kirsch, D. L. \& Lerner, F. N. (1998). Electromedicine. The other side of physiology. In: Weiner, R.S. (ed.). Innovations in pain management: a practical guide for clinician. Boca Raton (FL): St. Lucie Press.

[32]. Kirsch, D. L. \& Smith, R. B. (2000). The use of cranial electrotherapy stimulation in the management of chronic pain: a review. Neuro Rehabilitation, 14, 85-94.

[33]. Kirsch, D L. (1999). Post marketing survey of Alpha - Stim CES patients. In: Kirsch, D. L., editor. The science behind cranial electrotherapy stimulation. Edmonton (Canada): Medical Scope Publishing.

[34]. Smith, R. B.(1999). Cranial electrotherapy stimulation in the treatment of stress related cognitive dysfunction: with an eighteen month follow up. Journal of Cognitive Rehabilitation, 17, 14-18.

[35]. Overcash, S. J. (1999). A retrospective study to determine the effect of cranial electrotherapy stimulation (CES) on patients suffering from anxiety disorders. American Journal of Electromedicine, 16, 49-51.

[36]. Klawansky, S., Yeung, A., Berkey, C. Shan, N., Phan, H., \& Chalmers, T. C. (1995). Meta-analysis of randomized controlled trials of cranial electro stimulation. Efficacy in treating selected psychological and physiological conditions. Journal of Nervous and Mental Disease, 183, 478-485.

[37]. Bianco Jr., Faust.(1994) The efficacy of cranial electrotherapy stimulation (CES) for the relief of anxiety and depression among polysubstance abusers in chemical dependency treatment. Ph.D. dissertation. the University of Tulsa Graduate School,1994

[38]. Overcash, S. J. (1995). A retrospective study to determine the effect of cranial electrotherapy stimulation (CES) on patients suffering from anxiety disorders. Journal of Biofeedback and Self Regulation. (In press).

[39]. Avrahami, E. (1990). Panic attacks during MR imaging. American Journal of Neuroradiology, 11, 833 - 835.

[40]. Bystritsky, A., Kerwin, L. \&Feusner, J. (2009). A Pilot Study of Cranial Electrotherapy Stimulation for Generalsed Anxiety Disorder. Journal of Clinical Psychology, 69 (3), 412 - 417

[41]. Chavous, A. S. (1982). Biofeedback assisted relaxation and relaxation alone: Effects on headache symptoms, affect and cognitions Dissertation Abstracts International, 42, 20-86.

[42]. Hmud, R. \& Walsh, L. J. (2009). Dental anxiety: causes, complications and management approaches. Journal of Minimum Intervention in Dentistry, 2 (1), $67-77$

[43]. Humphris, G. M., and Morrison, T. and Lindsay, S. (1995). The Modified Dental Anxiety Scale: validation and United Kingdom norms. Community Dentistry and Oral Epidemiology, 12, $143-150$.

[44]. Klawansky, S. (1995). Meta-analysis of Randomized Controlled Trials of Cranial Electrostimulation: Efficacy in Treating Selected Psychological and Physiological Conditions. Journal of Nervous Mental Disorder, 183, 478-485.

[45]. Newton, J. T. \& Edwards, J. C. (2005). Psychometric properties of the modified dental anxiety scale: an independent replication. Community Dental Health, 22, 40-42

[46]. Overcash, S. J. (1995). A retrospective study to determine the effect of cranial electrotherapy stimulation (CES) on patients suffering from anxiety disorders. Journal of Biofeedback and Self Regulation. (In press).

[47]. Zaghi, S., Acar, M., Hultgren, B., Boggio, P. S. \&Fregni, F. (2009). Noninvasive Brain Stimulation with Low-Intensity Electrical Currents: Putative Mechanisms of Action for Direct and Alternating Current Stimulation. The Neuroscientist. 2010 Jun;16(3):285307 